Artículo científico

Volumen 32(2):523-537. Mayo-agosto, 2021

e-ISSN 2215-3608, doi:10.15517/am.v32i2.42862

https://revistas.ucr.ac.cr/index.php/agromeso/index

\title{
Producción y calidad de forraje de Sambucus nigra en cercas vivas, trópico alto colombiano ${ }^{1}$
}

\section{Production and quality of forage of Sambucus nigra in living fences, high Colombian tropics}

\author{
Alexander Navas-Panadero², Juan David Hernández-Larrota², Juan Carlos Velásquez-Mosquera²
}

1 Recepción: 3 de octubre, 2020. Aceptación: 10 de diciembre, 2020. Este trabajo es producto del proyecto "Contribución de las cercas vivas de tilo a la producción de forraje en sistemas ganaderos de trópico alto", financiado por la Universidad de La Salle.

2 Universidad de La Salle, Facultad de Ciencias Agropecuarias, Grupo de investigación REMEAT. Carrera No 179-03. Bogotá, Colombia. anavas@unisalle.edu.co (autor para correspondencia; https://orcid.org/0000-0001-8975-2601), juandhernandez00@unisalle.edu.co (https:// orcid.org/0000-0003-3431-4799), jcvelasquez@unisalle.edu.co (http://orcid.org/0000-0003-4066-5376).

\section{Resumen}

Introducción. La alimentación de los animales en los sistemas ganaderos del trópico alto colombiano se basa en el pastoreo de gramíneas en monocultivo de baja calidad y biomasa en los periodos de sequía, lo que afecta el desempeño productivo de los animales. Objetivo. Evaluar la producción y la calidad nutricional del forraje de Sambucus nigra L., establecido como un arreglo silvopastoril de cercas vivas. Materiales y métodos. La investigación se desarrolló en la finca San Joaquín, ubicada en Paipa, Colombia; los muestreos se realizaron entre enero 2019 y marzo 2020. En un arreglo de cercas vivas se seleccionó un transecto de $226 \mathrm{~m}$ lineales, en los que se escogieron al azar diecisiete árboles. Se evaluó la producción de forraje verde y materia seca de la planta completa y sus fracciones (hojas y tallos), la relación hoja - tallo y la calidad nutricional (NIRS) de la planta completa y de cada fracción. Se utilizó un diseño completamente al azar, se realizó estadística descriptiva y análisis de varianza no paramétrica (prueba de Kruskal Wallis) para producción de forraje y relación hoja-tallo, el análisis de los datos se hizo mediante el programa Infostat ${ }^{\circledR}$. Resultados. La producción de forraje verde y materia seca fue de $19,8 \mathrm{t} \mathrm{km}^{-1} \mathrm{año}^{-1}$ y $3,9 \mathrm{t} \mathrm{km}^{-1}$ $\mathrm{año}^{-1}$, respectivamente. Se presentaron diferencias $(\mathrm{p}<0,0001)$ en la calidad nutricional, las hojas presentaron mayor calidad, el forraje presentó altos contenidos de proteína cruda, energía metabolizable y digestibilidad, bajo porcentaje de fibra en detergente neutro y fibra en detergente ácido, la relación hoja-tallo fue de 0,8:1, aunque se presentaron diferencias entre muestreos $(\mathrm{p}<0,0001)$. Conclusiones. Las cercas vivas de Sambucus nigra generaron alta producción de biomasa comestible por kilómetro al año, el forraje de la planta completa y sus fracciones tuvieron buena calidad nutricional, especialmente proteína cruda, energía y digestibilidad.

Palabras claves: Saúco, sistemas silvopastoriles, alimentación, forraje, producción animal.

\begin{abstract}
Introduction. Livestock feeding in Colombian high tropic livestock systems is based on monoculture grass grazing of low quality and low biomass during drought periods, which affects the productive performance of the animals. Objective. To evaluate the production and nutritional quality of forage of Sambucus nigra L., established
\end{abstract}


as a silvopastoral live fence arrangement. Materials and methods. The research was carried out at the San Joaquin farm, located in Paipa, Colombia; the samplings were done between January 2019 and March 2020. In a live fence arrangement, a $226 \mathrm{~m}$ linear transect was selected, in which 17 trees were randomly selected. The green forage and dry matter production of the complete plant and its fractions (leaves and stems), the leaf - stem ratio and the nutritional quality (NIRS) of the complete plant and of each fraction were evaluated. A randomized design was carried out, descriptive statistics and analysis of non-parametric variance (Kruskal Wallis test) were performed for forage production and leaf-stem relationship, the data analysis was done using the Infostat ${ }^{\circledR}$ program. Results. The production of green forage and dry matter was $19, \mathrm{t} \mathrm{km}^{-1}$ year $^{-1}$ and $3,9 \mathrm{t} \mathrm{km}^{-1} \mathrm{year}^{-1}$, respectively. There were differences $(\mathrm{p}<0.0001)$ in nutritional quality, the leaves were of higher quality, the forage had a high crude protein content, metabolizable energy and digestibility, low percentage of neutral detergent fiber and acid detergent fiber, the leaf-stem ratio was 0.8: 1, although there were differences between samplings $(\mathrm{p}<0.0001)$. Conclusions. The living fences of S. nigra produced high production of edible biomass per kilometer per year, the forage of the complete plant and its fractions had good nutritional quality, crude protein, energy, and digestibility.

Keywords: Sambucus, silvopastoral systems, feeding, forage, animal production.

\section{Introducción}

Los sistemas de producción de leche en trópico alto (2000 a $3000 \mathrm{msnm})$ generan impactos ambientales negativos, en muchos casos se establecen en ecosistemas estratégicos como los páramos, causando degradación y perdida de servicios ecosistémicos importantes para la sociedad. Además, del uso de algunas tecnologías que pueden causar la pérdida del potencial productivo del suelo, la contaminación de aguas y la emisión de gases efecto invernadero (Estupiñán et al., 2009; Medina, 2016; Rivera et al., 2013a; Rousseau et al., 2013).

Los sistemas de producción convencionales utilizan monocultivos de pasturas y altas cantidades de insumos externos (agroquímicos, alimentos balanceados comerciales, ensilajes), esto incrementa los costos de producción y reduce la rentabilidad de las fincas, lo que puede generar problemas sociales y económicos en estos agroecosistemas.

La base de la alimentación en sistemas de producción ganaderos en el trópico alto es el monocultivo de especies de gramíneas como kikuyo [Centhrus clandestinum (Hochst. ex Chiov.)] y ryegrass (Lolium sp) (Vargas et al., 2018), estas presentan alta vulnerabilidad a condiciones climáticas extremas como épocas de sequía o heladas (Correa et al., 2018), lo que se refleja en la reducción de la producción y calidad nutricional de forraje, afectando la eficiencia productiva y reproductiva de los animales.

La producción de forraje a partir de especies arbóreas permite reducir el impacto en las épocas críticas y suplementar los animales durante los meses de abundancia de forraje en los potreros. La relación hoja-tallo es importante en las especies forrajeras, debido a que en las hojas presentan la mayor calidad nutricional, y la cantidad de estas presentes en la planta depende de varios factores como la calidad del suelo y las condiciones climáticas, algunas especies arbóreas forrajeras, en épocas de sequía botan las hojas para mantener el balance hídrico, afectando negativamente esta relación (Navas \& Montaña, 2019).

La calidad nutricional de la dieta es un factor importante en la expresión del potencial genético de los animales, la eficiencia productiva y reproductiva dependen en buena medida de que los aportes de nutrientes de los alimentos llenen los requerimientos nutricionales de los animales según su estado fisiológico. El valor nutricional de los forrajes está determinado en gran medida por las condiciones climáticas (luz, temperatura, humedad) y los factores de madurez, aunque la respuesta depende de cada especie (Van Soest, 1969).

La proteína cruda (PC) es un nutriente importante en la eficiencia productiva y reproductiva de los animales, comúnmente en sistemas de producción de leche debe ser suplementado, lo cual incrementa los costos de 
producción (Schöbitz et al., 2013). El forraje de especies arbóreas producido dentro de la finca puede reducir las necesidades de alimentos externos (Navas \& Montaña, 2019).

La composición química de un forraje está directamente relacionada con la edad de corte (Gómez et al., 2014), ya que con el aumento de la edad también se incrementan las fibras, lo que afecta negativamente el consumo voluntario y la concentración de energía disminuye (Weiss, 1993). Altas concentraciones de fibra en detergente ácido (FDA) se asocian con baja digestibilidad ruminal (Apráez et al., 2012; Van Soest et al., 2000), ya que, al ser parte de la pared celular, la lignina contenida en la FDA tiene una digestibilidad casi nula (Portillo et al., 2019). Por el contrario, bajos porcentajes de fibra en detergente neutro (FDN) permiten tener mayor contenido de componentes intracelulares (Villalobos \& Sánchez, 2010).

Los sistemas de lechería especializada también demandan altas cantidades de minerales para la producción, especialmente calcio $(\mathrm{Ca})$ y fósforo $(\mathrm{P})$. Durante el periodo de transición se pueden presentar enfermedades metabólicas (síndrome de vaca caída) por inadecuado manejo de los minerales, después del parto se hace necesario incrementar la cantidad para favorecer la producción y reproducción de las vacas.

Se deben establecer modelos de producción de ganadería sostenible, en los cuales se incorpore un manejo racional de los recursos, se reduzcan y/o elimine el uso de agroquímicos, se establezcan praderas bajo sistemas de pastoreo rotacional, se reduzcan la utilización de insumos, etc. Los sistemas silvopastoriles hacen parte de los sistemas productivos sostenibles, que mejoran las interacciones entre el suelo, la planta y el animal (Cárdenas et al., 2016; Escobar-Pachajoa et al., 2019), siendo de gran importancia en agroecosistemas de trópico alto (De-laOssa- Lacayo, 2013).

La mayoría de las fincas localizadas en el trópico alto colombiano presentan pequeñas áreas de pastoreo, los productores consideran que la siembra de árboles reduce la producción de pasto, por esta razón los sistemas silvopastoriles no han tenido mucha adopción en estos agroecosistemas, a pesar de que la siembra de árboles en sistemas de producción animal tenga múltiples beneficios (Frey et al., 2012).

Los sistemas silvopastoriles favorecen el mejoramiento del suelo a través del incremento de la materia orgánica y la actividad biológica, la conservación de la humedad, el ciclaje de nutrientes y la fijación de nitrógeno atmosférico que hacen algunas especies (Escobar et al., 2020; Rueda et al., 2011; Sánchez et al., 2007; Vallejo, 2013), lo que permite el incremento de la producción y calidad de las pasturas (Alonso, 2011; Navas et al., 2020; Rivera et al., 2013a). Además, los árboles a través de la sombra generan microclimas que permite a los animales mantenerse dentro o cerca de su zona confort (Esquivel, 2007; Navas, 2010), con efectos positivos sobre el consumo voluntario y la reducción del gasto energético para termoregularse (Córdova et al., 2009; Jarvis et al., 2010), lo que se refleja en el incremento de la producción leche (Cañas et al., 2003; Sousa et al., 2010) y el desempeño reproductivo (Souza, 2003), adicionalmente se reduce el uso de fertilizantes, concentrados y agroquímicos (Murgueitio et al., 2011).

Existen diversos arreglos silvopastoriles, el más común en sistemas de trópico alto son las cercas vivas, las cuales permiten la división de áreas de pastoreo. El sauco o tilo (Sambucus nigra) es una especie arbórea con potencial para establecer cercas vivas, ya que se adapta adecuadamente a condiciones ambientales del trópico alto de la región (Escobar-Pachajoa et al., 2019; Giraldo et al., 2011), además puede contribuir a la producción de forraje de buena calidad, aún en épocas críticas, ya que soporta condiciones de heladas (Grajales-Atehortúa et al., 2015).

El forraje de S. nigra es una alternativa nutricional para rumiantes, presenta alto contenido de proteína cruda (PC), baja cantidad de fibra, alta palatabilidad y digestibilidad (Cárdenas et al., 2011); estudios muestran en el forraje contenidos de $24 \%$ de PC (Cárdenas et al., 2016), 37,4 \% de fibra en detergente neutro (FDN) y 15,7 \% de fibra en detergente ácido (FDA) (Carvajal et al., 2012).

Por lo anterior, el objetivo de este estudio fue evaluar la producción y la calidad nutricional del forraje de $S$. nigra, establecido como arreglo silvopastoril de cercas vivas. 


\section{Materiales y métodos}

\section{Localización}

La investigación se desarrolló en la finca San Joaquín, dedicada a la lechería especializada, ubicada en el municipio de Paipa $\left(5^{\circ} 44^{\prime} 10^{\prime \prime} 73^{\circ} 04^{\prime} .43^{\prime \prime}\right)$, Boyacá, Colombia. A una altura de $2486 \mathrm{msnm}$, con temperatura promedio de $13,7^{\circ} \mathrm{C}$, precipitación media anual de $811 \mathrm{~mm}$ y humedad relativa de $78 \%$ (Instituto de Hidrología, Meteorología y Estudios Ambientales, s.f.).

El proyecto tuvo una duración de quince meses (enero de 2019 a marzo de 2020). Las características del suelo en el cual se trabajó presentaba topografía plana, del orden Andisol, con textura franco-arcillosa, pH de 4,01 y 12,5 $\%$ de materia orgánica. El sistema de producción se dedica a la lechería especializada, realiza pastoreo rotacional, el área de pastoreo estuvo dividida en potreros separados por cercas vivas de sauco (Sambucus nigra).

\section{Diseño experimental}

Se estableció el ensayo en un arreglo silvopastoril de cercas vivas con más de trece años de establecidas, con una longitud de $4 \mathrm{~km}$, con árboles sembrados a una distancia de 1,50 m entre ellos. Se seleccionó un transecto de $226 \mathrm{~m}$ lineales, en el cual los árboles presentaron en promedio $17,3 \mathrm{~cm}$ de diámetro a la altura del pecho (DAP), 5,3 m de altura total y $37,7 \mathrm{~m}^{2}$ de área de copa. En el transecto se seleccionaron al azar diecisiete árboles para evaluar la producción de forraje de la planta completa y cada fracción (hojas y tallos comestibles), a los cuales se les realizó un corte de homogenización a 1,30 m del suelo, este corte también se les realizó a los árboles que estaban a los lados para evitar efecto de sombreo sobre los árboles de la evaluación. Se utilizó un diseño completamente al azar. Transcurrido el tiempo de rebrote (90 días) se iniciaron los muestreos. La cerca viva no tuvo ningún tipo de fertilización, ni otro tipo de manejo.

\section{Variables evaluadas}

La producción de forraje verde (FV) del árbol completo y sus fracciones (hojas y tallos) se determinó mediante cosecha manual de los diecisiete árboles. Se realizaron cinco cortes, cada corte se efectuó cuando los árboles tuvieron noventa días de recuperación. Los árboles se podaron a una altura de 1,30 m del suelo y cada individuo se pesó (balanza digital) por separado. Posteriormente, cada planta se fraccionó manualmente y se pesaron las hojas y tallos por separado para determinar la proporción y relación de biomasa entre las fracciones (hoja-tallo), con base en esta información se extrapoló la producción de la cerca ( $\mathrm{t} \mathrm{km}{ }^{-1}$ corte)

Para determinar la calidad nutricional se tomaron muestras de $500 \mathrm{~g}$ de FV de la planta completa (conformada por hojas y tallos comestibles) y lo mismo que para hojas y tallos por separado. Estas se llevaron al laboratorio, mediante metodología de espectrofotometría de infrarrojo cercano (NIRS), se les determinó materia seca (MS), proteína cruda (PC), fibra en detergente neutro (FDN), fibra en detergente ácido (FDA), digestibilidad, energía neta de lactancia $(\mathrm{ENl})$, cenizas, calcio $(\mathrm{Ca})$, fósforo $(\mathrm{P})$, magnesio $(\mathrm{Mg})$ y potasio $(\mathrm{K})$.

Se registró la precipitación acumulada previa a cada corte, la cual correspondió a los días de recuperación de los árboles entre cortes (noventa días), los datos se tomaron con un pluviómetro instalado en la finca.

\section{Análisis estadístico}

Se realizó un ANOVA y tukey para las variables producción de forraje verde y materia seca de la planta completa y sus fracciones (hojas y tallos comestibles), relación hoja-tallo y de calidad nutricional. El análisis de los datos se realizó mediante el programa Infostat ${ }^{\circledR}$ (Di-Rienzo et al., 2018). 


\section{Resultados}

\section{Comportamiento de la precipitación}

Durante el periodo experimental se observaron diferencias en la cantidad de precipitación acumulada antes de cada uno de los cinco cortes (Figura 1), hubo mayor precipitación en el corte tres, seguido del cinco, mientras que los cortes uno, dos y cuatro tuvieron menor cantidad de agua, lo que posiblemente pudo afectar las variables en estudio.

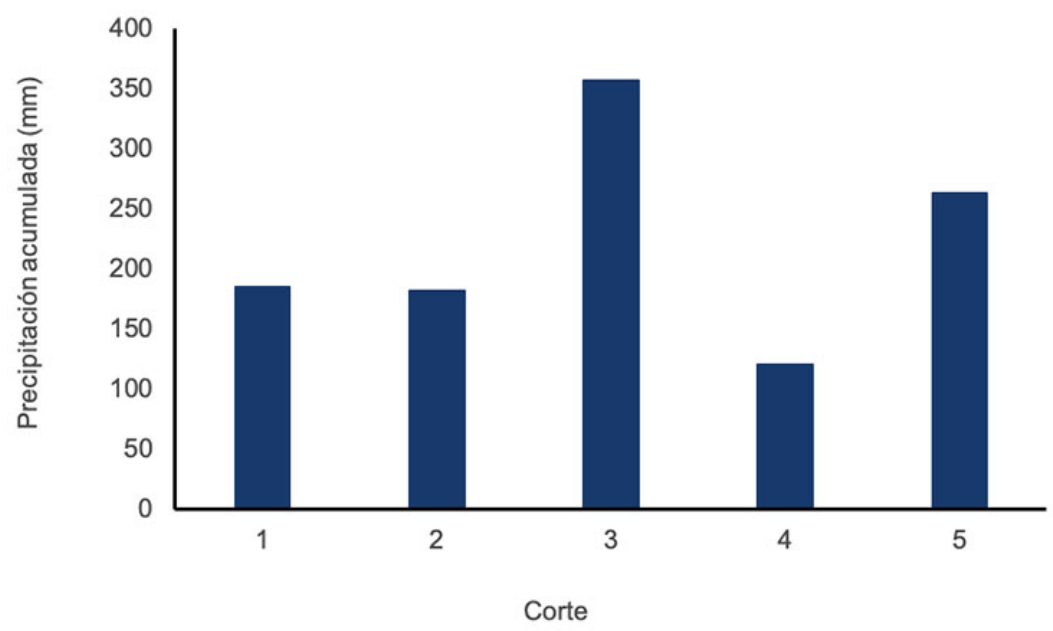

Figura 1. Comportamiento de la precipitación acumulada (noventa días) antes de cada corte, durante la evaluación de cercas vivas de Sambucus nigra, en Paipa, Colombia. 2019.

Figure 1. Behavior of the accumulated precipitation (ninety days) before each cut, during the evaluation of Sambucus nigra living fences, in Paipa, Colombia. 2019.

\section{Producción de forraje verde y materia seca}

La producción de forraje verde (FV) y materia seca (MS) de S. nigra encontrada demostró que puede ser una alternativa de producción de biomasa importante en sistemas ganaderos. La producción promedio de FV por planta/ corte fue de 7,4 kg, y fue ligeramente mayor la producción de tallos que de hojas (Cuadro 1). Según los resultados encontrados esta especie puede producir 19,8 $\mathrm{t} \mathrm{km}^{-1}$ y $3,9 \mathrm{t} \mathrm{km}^{-1}$ de FV y MS al año, respectivamente.

Se presentaron diferencias $(\mathrm{p}<0,0001)$ entre cortes en la producción de MS para la planta completa, hojas y tallos (Figura 2). El corte dos presentó mayor producción de la planta completa $\left(1,76 \mathrm{t} \mathrm{km}^{-1}\right)$, seguido por el corte cinco $\left(1,01 \mathrm{t} \mathrm{km}^{-1}\right)$, mientras que la menor producción la presentó el corte uno $\left(0,45 \mathrm{t} \mathrm{km}^{-1}\right)$. En la mayoría de los cortes se presentó mayor producción de tallos con relación a las hojas, excepto en el corte tres, donde la producción de hojas fue ligeramente superior.

Se encontró una relación hoja-tallo promedio de 0,8:1, aunque se presentaron diferencias entre muestreos $(\mathrm{p}<0,0001)$, siendo menor la relación en los muestreos cuatro $(0,7: 1)$ y cinco $(0,7: 1)$, mientras que la mayor relación se presentó en los muestreos dos $(1,1: 1)$ y tres $(0,9: 1)$, los cuales fueron precedidos por mayor precipitación (Figura 3). 
Cuadro 1. Producción promedio (por corte) de biomasa de la planta completa y de cada fracción (hoja, tallo), en forraje verde y materia seca de Sambucus nigra en cercas vivas, cada noventa días, con podas a una altura de 1,30 m del suelo, en Paipa, Colombia. 2019.

Table 1. Average production (per cut) of biomass of the complete plant and of each fraction (leaf, stem), in green forage and dry matter of Sambucus nigra in living fences, every ninety days, with pruning at a height of $1.30 \mathrm{~m}$ of height from the ground, in Paipa, Colombia. 2019.

\begin{tabular}{|c|c|c|c|c|}
\hline \multirow{3}{*}{$\begin{array}{l}\text { Fracción de la planta } \\
\text { Planta completa }\end{array}$} & Producción de FV & Producción de MS & Producción de FV & Producción de MS \\
\hline & \multicolumn{2}{|c|}{ (g/planta/corte) } & \multicolumn{2}{|c|}{ (t km-1/corte) } \\
\hline & $7405 \pm 406$ & $1477 \pm 95$ & $4,94 \pm 0,3$ & $0,98 \pm 0,06$ \\
\hline Hojas & $3325 \pm 206$ & $632 \pm 33$ & $2,22 \pm 0,1$ & $0,42 \pm 0,02$ \\
\hline Tallos & $4018 \pm 219$ & $904 \pm 58$ & $2,68 \pm 0,1$ & $0,60 \pm 0,04$ \\
\hline
\end{tabular}

\pm error estándar. FV: forraje verde. MS: materia seca. 667 árboles $\mathrm{km}^{-1} / \pm$ standard error. FV: green fodder. MS: dry matter. 667 trees $\mathrm{km}^{-1}$.

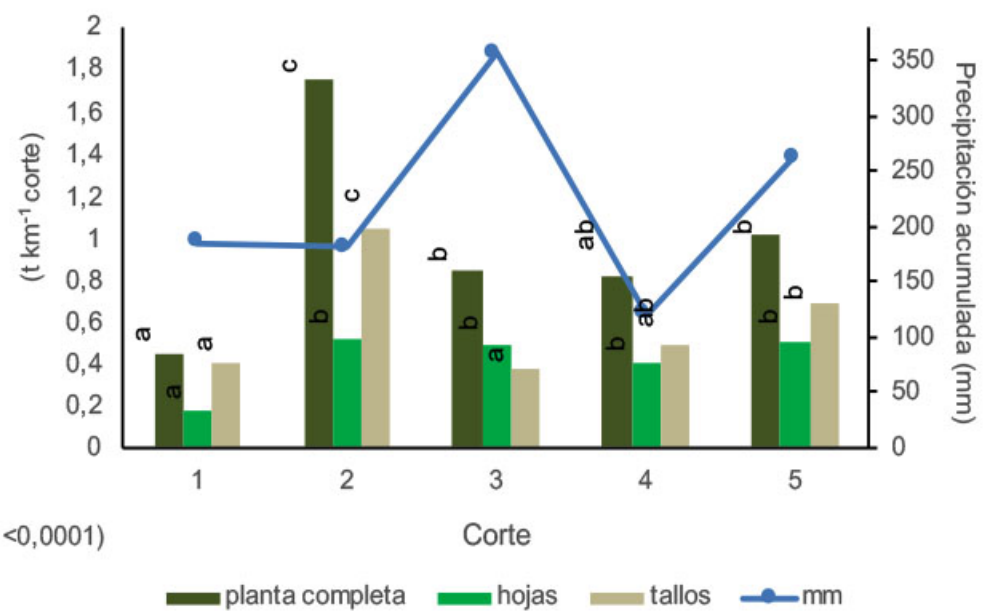

Figura 2. Producción de materia seca ( $\mathrm{km}^{-1} / \mathrm{corte}$ ) de la planta completa y de cada fracción (hoja, tallo), de Sambucus nigra, en cercas vivas, y precipitación acumulada antes del corte, cada noventa días, con podas a una altura de 1,30 m del suelo, en Paipa, Colombia. 2019. Medias con una letra común no son significativamente diferentes $(\mathrm{p}>0,05)$.

Figure 2. Dry matter ( $\mathrm{t} \mathrm{km}^{-1} / \mathrm{cut}$ ) production of the complete plant and of each fraction (leaf, stem), of Sambucus nigra in living fences, and accumulated precipitation before the cut, every ninety days, with pruning at $1.30 \mathrm{~m}$ of height from the ground, in Paipa, Colombia. 2019. Means with a common letter are not significantly different $(\mathrm{p}>0.05)$.

\section{Calidad nutricional de la planta completa y fracciones (hoja-tallo)}

La calidad nutricional del forraje de la planta completa de $S$. nigra y de sus fracciones (hoja, tallo) se puede considerar superior a las gramíneas forrajeras de la zona (Cuadro 2), ya que presentó un elevado porcentaje de proteína cruda (PC), energía neta de lactancia (ENl) y digestibilidad, mientras que los contenidos de fibra en detergente neutro (FDN) y fibra en detergente ácido (FDA) fueron bajos. Las hojas fueron la fracción de la planta que presentó mayores contenidos y mejor calidad nutricional, se encontraron diferencias estadísticas $(\mathrm{p}<0,0001)$ en todas las variables con relación a los tallos. 


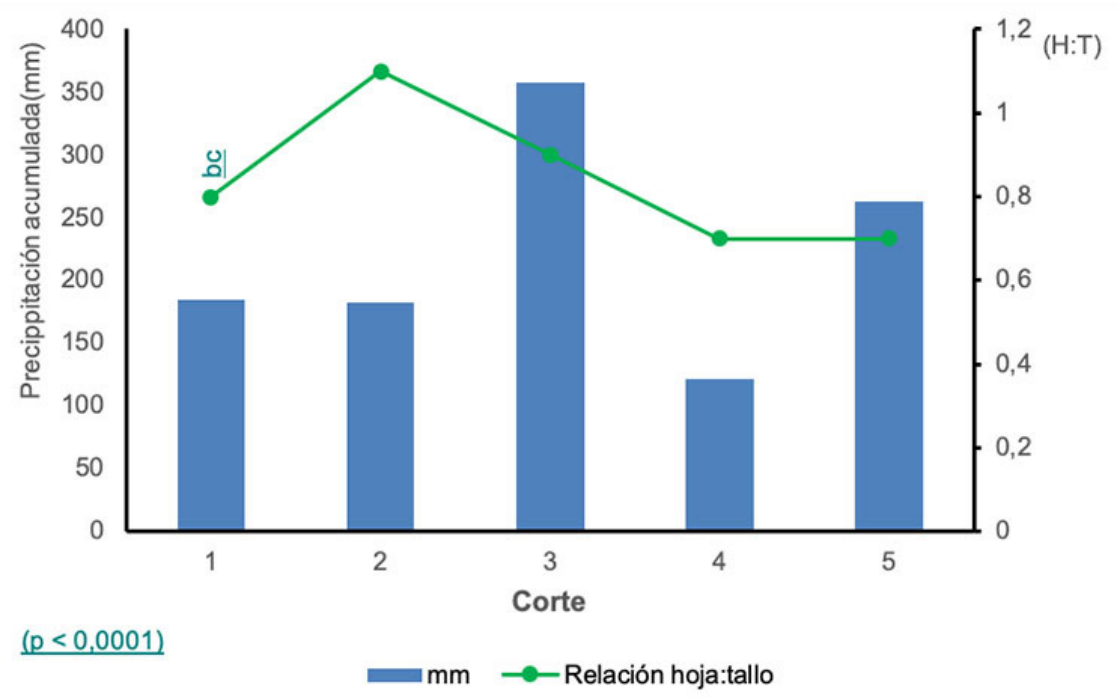

Figura 3. Relación hoja-tallo de plantas de Sambucus nigra en cercas vivas y precipitación acumulada antes de cada corte, cada noventa días, con podas a una altura de 1,30 m del suelo, en Paipa, Colombia. 2019.

Figure 3. Leaf-stem relationship of Sambucus nigra plants in living fences and accumulated precipitation before each cut, every ninety days, with pruning at $1.30 \mathrm{~m}$ of height from the ground, in Paipa, Colombia. 2019.

Cuadro 2. Calidad nutricional de la planta completa y de cada fracción (hoja-tallo) de Sambucus nigra en cercas vivas, cada noventa días, con podas a una altura de 1,30 m del suelo, en Paipa, Colombia. 2019.

Table 2. Nutritional quality of the complete plant and of each fraction (leaf-stem) of Sambucus nigra in living fences, every ninety days, with pruning at $1.30 \mathrm{~m}$ of height from the ground, in Paipa, Colombia. 2019.

\begin{tabular}{lcccccc}
\hline Fracción de la planta & MS $(\%)$ & PC $(\%)$ & digest $(\%)$ & ENI $\left(\mathbf{M c a l ~ k g}^{-1}\right)$ & FDN $(\%)$ & FDA $(\%)$ \\
\hline Planta completa & $19,7 \pm 1 \mathrm{a}$ & $23,7 \pm 2 \mathrm{~b}$ & $72,6 \pm 2 \mathrm{~b}$ & $1,5 \pm 0,1 \mathrm{~b}$ & $35,6 \pm 3 \mathrm{a}$ & $18,7 \pm 3 \mathrm{a}$ \\
Hojas & $20,0 \pm 2 \mathrm{a}$ & $28,5 \pm 1 \mathrm{~b}$ & $77,9 \pm 1 \mathrm{~b}$ & $1,6 \pm 0,1 \mathrm{~b}$ & $28,8 \pm 3 \mathrm{a}$ & $13,9 \pm 3 \mathrm{a}$ \\
Tallos & $23,7 \pm 4 \mathrm{a}$ & $11,7 \pm 1 \mathrm{a}$ & $57,7 \pm 2 \mathrm{a}$ & $1,2 \pm 0,1 \mathrm{a}$ & $57,4 \pm 3 \mathrm{~b}$ & $35,3 \pm 3 \mathrm{~b}$ \\
$\mathrm{p}$ & 0,4810 & $<0,0001$ & $<0,0001$ & $<0,0001$ & $<0,0001$ & 0,0011 \\
\hline
\end{tabular}

MS: materia seca; PC: proteína cruda; Digest: digestibilidad; ENl: energíaneta de lactancia; FDN: fibra en detergente neutro; FDA: fibra en detergente ácido. \pm : error estándar. Medias con una letra común en la columna no son significativamente diferentes ( $\mathrm{p}>0,05)$ / MS: dry matter; PC: crude protein; Digest: digestibility; NEL: net energy of lactation; FDN: fiber in neutral detergent; FDA: fiber in acid detergent. \pm : standard error. Means with a common letter in the column are not significantly different $(\mathrm{p}>0.05)$.

En cuanto al contenido de minerales se encontró que S. nigra presentó mayores contenidos de calcio, fósforo, magnesio, potasio y azufre que las gramíneas forrajeras de la zona (Cuadro 3). No se encontraron diferencias entre planta completa y cada una de las fracciones en los contenidos de los minerales evaluados, con excepción del magnesio $(\mathrm{p}=0,0008)$, el cual presentó menores contenidos en los tallos. 
Cuadro 3. Contenido de minerales de la planta completa y de cada fracción (hoja-tallo) de Sambucus nigra en cercas vivas, cada noventa días, con podas a una altura de 1,30 m del suelo, en Paipa, Colombia. 2019.

Table 3. Mineral content of the complete plant and of each fraction (leaf-stem) of Sambucus nigra in living fences, every ninety days, with pruning at $1.30 \mathrm{~m}$ of height from the ground, in Paipa, Colombia. 2019.

\begin{tabular}{lccccc}
\hline Fracción de la planta & Cenizas $(\boldsymbol{\%})$ & Ca $(\boldsymbol{\%})$ & $\mathbf{P}(\boldsymbol{\%})$ & $\mathbf{M g}(\boldsymbol{\%})$ & $\mathbf{K}(\boldsymbol{\%})$ \\
\hline Planta completa & $10,2 \pm 0,4 \mathrm{~b}$ & $0,70 \pm 0,1 \mathrm{a}$ & $0,26 \pm 0,03 \mathrm{a}$ & $0,30 \pm 0,02 \mathrm{~b}$ & $2,43 \pm 0,2 \mathrm{a}$ \\
Hojas & $10,5 \pm 0,3 \mathrm{~b}$ & $0,63 \pm 0,1 \mathrm{a}$ & $0,32 \pm 0,02 \mathrm{a}$ & $0,30 \pm 0,02 \mathrm{~b}$ & $2,36 \pm 0,2 \mathrm{a}$ \\
Tallos & $6,6 \pm 0,6 \mathrm{a}$ & $0,52 \pm 0,02 \mathrm{a}$ & $0,25 \pm 0,02 \mathrm{a}$ & $0,19 \pm 0,02 \mathrm{a}$ & $2,30 \pm 0,2 \mathrm{a}$ \\
$\mathrm{p}$ & 0,0001 & 0,4674 & 0,1510 & 0,0008 & 0,9016 \\
\hline
\end{tabular}

Ca: calcio; P: fósforo; Mg: magnesio; K: potasio; S: azufre. \pm : error estándar. / Ca: calcium; P: phosphorus; Mg: magnesium; K: potassium; S: sulphur. \pm : standard error.

\section{Discusión}

La producción forraje de S. nigra se ha determinado en bancos forrajeros, pero no en cercas vivas. Este estudio presentó mayor producción de forraje verde $\left(19,8 \mathrm{t} \mathrm{km}^{-1} / \mathrm{año}\right)$ y materia seca $\left(3,9 \mathrm{t} \mathrm{km}^{-1} / \mathrm{año}\right)$ que la encontrada en otros estudios (16,5 $\mathrm{t} \mathrm{ha}^{-1} / \mathrm{año}$ y $2,44 \mathrm{t} \mathrm{ha}^{-1} / \mathrm{año}$, respectivamente), pero en bancos forrajeros (González-Guarín, 2016) este comportamiento también se reportó en trabajos que evaluaron solo la producción de forraje verde y donde se mencionaron 15,2 $\mathrm{t} \mathrm{ha}^{-1} /$ año y 12,7 t ha-1/año (Apráez et al., 2012; Cárdenas et al., 2016), lo que demuestra el potencial de la especie en arreglos de cerca viva, los cuales son preferidos por los productores en la zona de estudio.

Se presentó mayor producción de forraje verde y materia seca por arbusto $(7,4 \mathrm{~kg} /$ corte y $1,4 \mathrm{~kg} / \mathrm{corte}$, respectivamente), que lo encontrado en otros estudios $(2,03 \mathrm{~kg} /$ corte y $0,36 \mathrm{~kg} /$ corte, respectivamente) donde se cosecho el forraje a la misma edad de recuperación, pero en bancos forrajeros (Guatusmal-Gelpud et al., 2020), esto se puede explicar posiblemente por una mayor competencia por recursos que se puede presentar entre arbustos en los bancos forrajeros (Gómez et al., 2002), al igual que por condiciones climáticas durante el desarrollo de los estudios (Portillo et al., 2019).

La diferencia en la producción de forraje verde y materia seca entre cortes se puede atribuir principalmente a las condiciones climáticas durante el periodo de recuperación de los arbustos previos a los cortes (Portillo et al., 2019), se observó que la mayor producción se presentó cuando la precipitación acumulada estuvo alrededor de los 200 mm, mientras que la alta precipitación pareció afectar la producción de los arbustos; en ese sentido, los cortes uno y cinco se realizaron en enero, mes en el cual se presentaron eventos extremos de heladas, lo que pudo afectar la producción de forraje. La reducción en la producción que se presentó en el corte cuatro se puede atribuir a una defoliación de los arbustos por parte de las vacas al perderse la energía de la cerca eléctrica. Además, la baja producción que se presentó en el primer corte se puede deber en parte a la poda drástica de las plantas que estaban a libre crecimiento y a la cuales se les inició un nuevo manejo, lo que generó cambios en la planta, la cual incrementó con los cortes la cantidad de yemas de crecimiento y ramas (Gómez et al., 2002).

Este trabajo encontró menor relación hoja:tallo $(0,8: 1)$ que la reportada $(8,4: 1)$ en estudios realizados en arreglos de banco forrajero (Carvajal-Salcedo \& Cuesta-Peralta, 2016), lo que se puede explicar en gran medida por diferencias metodológicas, ya que el estudio realizado en bancos consideró los peciolos dentro de las hojas, incrementando esta fracción de la planta. Esta diferencia se puede atribuir en parte al manejo de los arbustos, en el estudio de los bancos las plantas desde el inicio recibieron cortes periódicos, mientras que en este estudio los arbustos en la cerca viva presentaron libre crecimiento durante 13 años, luego se podaron drásticamente y tres 
meses después se inició la evaluación, por lo tanto, las plantas tuvieron menor número de yemas de crecimiento y ramas, lo que pudo reducir la relación hoja-tallo (Gómez et al., 2002). Además, durante el cuarto corte la relación hoja-tallo se afectó negativamente por la defoliación que hicieron las vacas al dañarse la cerca eléctrica.

Para el caso de $S$. nigra se encontraron contenidos de MS en la planta completa diferentes a este estudio (19,7 $\%$ ), algunos fueron mayores 22,5 \% y 22,6 \% (Carvajal-Salcedo \& Cuesta-Peralta, 2016; Sánchez et al., 2010, respectivamente), mientras que otros estudios reportaron resultados similares 19,6, 19, 20,1, y 18,9\% (Apráez et al., 2012; Bohada-Hurtado et al., 2017; Fonseca-López et al., 2019; Rivera et al., 2013b, respectivamente).

La diferencia del porcentaje de MS se puede explicar posiblemente por diferencias en las condiciones climáticas durante los estudios, especialmente la precipitación (Larbi et al., 2010; Portillo et al., 2019), siendo mayor bajo condiciones de sequía o baja precipitación. También se puede explicar por la edad de recuperación de los arbustos (algunos estudios presentaron cortes a 120 días), llegando a alcanzar valores entre 38,5\% y 43,8\% a un mayor tiempo de recuperación (Cárdenas et al., 2016).

Los tallos fueron la fracción de la planta con mayor contenido de MS (23,7\%), lo que corresponde con lo mencionado en otros estudios, aunque el porcentaje fue mayor (26,9\%) (Carvajal-Salcedo \& Cuesta-Peralta, 2016; Sánchez et al., 2010).

El forraje de S. nigra presentó alto porcentaje de PC en la planta completa (23.7\%), aunque estudios similares han reportado contenidos mayores con $25,7 \%$ (Rivera et al., 2013a) y otros porcentajes menores entre 13,4\% y 20,9 \% (Bohada-Hurtado et al., 2017; Carvajal-Salcedo \& Cuesta-Peralta, 2016; Fonseca-López et al., 2019; Guatusmal-Gelpud et al., 2020; Sánchez et al., 2010), en todos los casos se observó que esta especie tiene alto potencial para ser utilizada en sistemas de lechería especializada por su contenido de PC.

Las hojas fueron la fracción de la planta con mayor porcentaje de PC $(28,5 \%)$, otros estudios reportaron menor contenido en las hojas (18,7 \%) (Carvajal-Salcedo \& Cuesta-Peralta, 2016; Sánchez et al., 2010). Los tallos (11,7\%) presentaron la misma tendencia en estos estudios que informaron valores de 9,3\% (Carvajal-Salcedo \& CuestaPeralta, 2016; Sánchez et al., 2010).

Las diferencias en el porcentaje de PC se pueden atribuir a los aportes de materia orgánica natural que han hecho los animales (orina y heces) bajo la copa de los árboles cuando hacen uso de la sombra, también al reciclaje que hacen los árboles recuperando nutrientes de perfiles profundos del suelo a través de su sistema radicular y acumulándolos en su follaje (Escobar et al., 2020; Sánchez et al., 2007; Vallejo, 2013).

La digestibilidad de un forraje es muy importante, debido a que representa el grado de aprovechamiento de los nutrientes por parte del animal, a mayor digestibilidad mayor expresión del potencial genético de los animales. La digestibilidad encontrada en la planta completa de $S$. nigra fue buena (72,6\%), siendo mayor en las hojas (77,9 $\%)$. Estudios han reportado similar digestibilidad en la planta completa (72,5\% con noventa días de recuperación) o mayores con 76,3 \% cuando el periodo de recuperación fue de sesenta días (Guatusmal-Gelpud et al., 2020), mientras que otros encontraron menor porcentaje (entre 52,7 \% y 66,6 \%) (Ayala-Russi et al., 2015; GonzálezGuarín, 2016; Rivera et al., 2013b).

El comportamiento de la digestibilidad se puede explicar por el tiempo de recuperación de los arbustos, las especies forrajeras tienen mejor digestibilidad cuando el forraje es más joven y se reduce conforme se incrementa la edad (Van Soest et al., 2000), aunque la reducción en especies arbóreas forrajeras no es tan rápida como se observa en especies gramíneas (Gómez et al., 2002). El suministro de forraje de $S$. nigra se puede reflejar en el incremento de la eficiencia productiva sin condicionar el consumo voluntario de los animales (Rivera et al., 2013b).

En sistemas de lechería especializada de trópico alto la energía es quizá el nutriente que puede limitar la producción y reproducción de los animales por desequilibrio en la relación energía - proteína, especialmente en la primera fase de lactancia, donde las vacas presentan balance energético negativo (Portillo et al., 2019). El forraje de la planta completa de $S$. nigra presentó altos contenidos energía neta de lactancia (1,5 $\left.\mathrm{Mcal} \mathrm{kg}^{-1}\right)$, siendo mayor en las hojas $\left(1,6 \mathrm{Mcal} \mathrm{kg}^{-1}\right)$, resultados que estuvieron por encima de los aportes energéticos de las principales 
gramíneas utilizadas en los potreros de estos agroecosistemas (Portillo et al., 2019), lo que convierte a S. nigra en un recurso forrajero importante para el manejo nutricional de los sistemas de lechería especializada. Estudios realizados reportaron menor contenido de energía metabolizable (EM) en la planta completa $\left(1,96 \mathrm{Mcal} \mathrm{kg}^{-1}\right)$ (González-Guarín, 2016) que la encontrada en este estudio (2,5 Mcal kg-1).

Los niveles de FDN y FDA obtenidas en el forraje de $S$. nigra fueron bajos, características que favorecen el aprovechamiento de los nutrientes a nivel ruminal y el consumo voluntario de los animales. Varios estudios encontraron en la planta completa niveles de FDN inferiores (entre 23,1 \% y 28,6 \%) (Apráez et al., 2012; FonsecaLópez et al., 2019; Guatusmal-Gelpud et al., 2020; Rivera et al., 2013b), al igual que en hojas (18,5\%) y tallos (20,3 \%) (Jaramillo, 2019), con relación a lo mencionado en este estudio de 35,6 \%, 28,8 \% y 57,4 \% para planta completa, hojas y tallos, respectivamente.

La FDA encontrada en la planta completa por otros estudios fue menor (13,8 \%, 15,7 \% y 13,9 \%) (BohadaHurtado et al., 2017; Carvajal et al., 2012; Guatusmal-Gelpud et al., 2020, respectivamente), a la reportada en este trabajo $(18,7 \%)$, mientras que otros autores mencionaron mayores contenidos $(21,1 \%, 31,8 \%, 34,8 \%$ y 21,1 \%) (Ayala-Russi et al., 2015; Cárdenas et al., 2016; Fonseca-López et al., 2019; Tabla-Rojas, 2019). Este comportamiento se puede atribuir al mayor tiempo de recuperación de los arbustos (Cárdenas et al., 2016; Gómez et al., 2014), y a posibles diferencias en las condiciones edafoclimáticas (Larbi et al., 2010; Portillo et al., 2019; Van Soest, 1969).

El contenido de cenizas encontrado en la planta completa por varios estudios fue superior $(11,0 \%, 12,9$ \% y 12,3 \%) (Bohada-Hurtado et al., 2017; Cárdenas et al., 2016; González-Guarín, 2016), mientras que otros reportaron valores similares $(9,8 \%$ y 10,1\%) (Carvajal-Salcedo \& Cuesta-Peralta, 2016; Fonseca-López et al., 2019 , respectivamente) a lo encontrado en este estudio $(10,2 \%)$.

Se observaron diferencias en contenidos de varios minerales en la planta completa con otros estudios, se reportó mayor contenido de Ca $(0,84 \%$ y $2,54 \%)$, menor de P $(0,21 \%$ y $0,24 \%)$ (Guatusmal-Gelpud et al., 2020; Rivera et al., 2013a, respectivamente), mayor de $\operatorname{Mg}(0,99 \%$ y 0,61 \%) (González-Guarín, 2016; Jaramillo, 2006), menor de K (2,25 \%) (González-Guarín, 2016); mientras que otro estudio mencionó mayor porcentaje (3,70 $\%)$ para este último mineral (Jaramillo, 2006), con relación a lo encontrado en este trabajo. La diferencia en el contenido de cenizas y minerales se puede explicar por las diferencias edáficas o la fertilización que realizaron en los estudios de los bancos forrajeros (Portillo et al., 2019).

\section{Conclusión}

Las cercas vivas de $S$. nigra generaron alta producción de biomasa comestible por $\mathrm{km}$ al año, el forraje de la planta completa y sus fracciones tuvieron buena calidad nutricional, especialmente las hojas, sobresale la proteína cruda, la energía y la digestibilidad. Este sistema silvopastoril puede contribuir a reducir la estacionalidad de la producción de forraje que se presenta en los sistemas ganaderos del trópico alto, además podría reducir la necesidad de insumos externos para suplementar a los animales.

\section{Agradecimientos}

Los autores agradecen a la Universidad de La Salle por la financiación del estudio. 


\section{Referencias}

Alonso, J. (2011). Los sistemas silvopastoriles y su contribución al medio ambiente. Revista Cubana de Ciencia Agrícola, 45(2), 107-115. https://www.redalyc.org/pdf/1930/193022245001.pdf

Apráez, J. E., Delgado, J. M., \& Narváez, J. P. (2012). Composición nutricional, degradación in vitro y potencial de producción de gas, de herbáceas, arbóreas y arbustivas encontradas en el trópico alto de Nariño. Livestock Research for Rural Development, 24(3), Artículo 24044. http://www.lrrd.org/lrrd24/3/apra24044.htm

Ayala-Russi, A. M., Rincón-Camacho, J. C., Navas-Panadero, A., \& González-Guarín, J. (2015). Evaluación de la selectividad de especies arbóreas con potencial forrajero en bovinos en ecosistemas de bosque húmedo premontano. Revista Ciencia Animal, 9, 41-55. https://ciencia.lasalle.edu.co/ca/vol1/iss9/4/

Bohada-Hurtado, C. M., Ospina, L. A., \& Vargas Sánchez, J. E. (2017). Identificación y caracterización de especies vegetales con potencial forrajero en trópico alto de la cuenca del rio tapias. Livestock Research for Rural Development, 29(5), Artículo. 29100 https://lrrd.cipav.org.co/lrrd29/5/karm29100.html

Cañas, R., Quiroz, R., Leon-Velarde, C., Posadas, A., \& Osorio, J. (2003). Quantifying energy dissipation by grazing animals in harsh environments. Journal of Theoretical Biology, 225, 351-359. https://doi.org/10.1016/S0022-5193(03)00260-1

Cárdenas, C. A., Rocha, C., \& Mora Delgado, J. (2011). Productividad y preferencia de forraje de vacas lecheras pastoreando un sistema silvopastoril intensivo de la zona alto Andina de Roncevalles, Tolima. Revista Colombiana de Ciencia Animal, 4(2011), 29-35. http://revistas.ut.edu.co/index.php/ciencianimal/article/view/140

Cárdenas, C. A., Rocha, C., \& Castañeda, R. D. (2016). Efecto de la edad de corte sobre las medidas morfométricas, la composición bromatológica y el fraccionamiento de la proteína del saúco (Sambucus nigra). Agronomía Costarricense, 40(2), 107-116. https://doi.org/10.15517/RAC.V40I2.27390

Carvajal, T., Lamela, L., \& Cuesta, A. (2012). Evaluación de las arbóreas S. nigra y Acacia decurrens como suplemento para vacas lecheras en la Sabana de Bogotá, Colombia. Pastos y Forrajes, 35(4), 417-430. https://www.researchgate. net/publication/262664549_Evaluacion_de_las_arboreas_Sambucus_nigra_y_Acacia_decurrens_como_suplemento_ para_vacas_lecheras_en_la_Sabana_de_Bogota_Colombia

Carvajal-Salcedo, T., \& Cuesta-Peralta, A. (2016). Conservación y composición nutricional del follaje de sauco (Sambucus nigra). Pastos y Forrajes, 39(2), 125-132. https://www.researchgate.net/publication/317513696_Conservacion_y_ composicion_nutricional_del_follaje_de_sauco_Sambucus_nigra

Córdova, A., Murillo, A., \& Castillo, H. (2009). Efecto de factores climáticos sobre la conducta reproductiva bovina en los trópicos. Revista Electrónica de Veterinaria, 11(1), 1-12. https://www.redalyc.org/pdf/636/63613103016.pdf

Correa, H. J., Escalante, L. F., \& Jaimes, L. J. (2018). Efecto de la época del año y la altura remanente posterior al pastoreo sobre el crecimiento y calidad nutricional del pasto kikuyo (Cenchrus clandestinus) en el norte de Antioquia. Livestock Research for Rural Development, 30, Artículo 97. http://www.lrrd.org/lrrd20/4/corra20059.htm

De-la-Ossa- Lacayo, A. (2013). Cercas vivas y su importancia ambiental en la conservación de avifauna nativa. Revista Colombiana de Ciencia Animal, 5(1), 171-193. https://doi.org/10.24188/recia.v5.n1.2013.483

Di-Rienzo, J., Casanoves, F., Balzarina, M., González, L., Tablada, M., \& Robledo, C. (2018). Infostat (versión 2018) [software de computadora]. Universidad Nacional de Córdoba. https://www.infostat.com.ar/ 
Escobar, M., Navas, A., Medina, C., Corrales, J., Tenjo, \& A., Borrás, L. (2020). Efecto de prácticas agroecológicas sobre características del suelo en un sistema de lechería especializada del trópico alto colombiano. Livestock Research for Rural Development, 32(4), Artículo 38. http://www.lrrd.org/lrrd32/4/maria.es32058.html

Escobar-Pachajoa, L. D., Guatusmal-Gelpud, C., Meneses-Buitrago, D. H., Cardona-Iglesias, J. L., \& Castro-Rincón. (2019). Evaluación de estratos arbóreos y arbustivos en un sistema silvopastoril en el trópico altoandino colombiano. Agronomía Mesoamericana, 30(3), 803-819. https://doi.org/10.15517/am.v30i3.35645

Esquivel, H. (2007). Tree resources in traditional silvopastoral systems and their impact on productivity and nutritive value of pastures in the dry tropics of Costa Rica [Tesis de Doctorado, Centro Agronómico Tropical de Investigación y Enseñanza]. Repositorio del Centro Agronómico Tropical de Investigación y Enseñanza. http://orton.catie.ac.cr/ repdoc/A1656e/A1656e.pdf

Estupiñán, L., Gómez, J., Barrantes, V., \& Limas, L. (2009). Efecto de actividades agropecuarias en las características del suelo en el páramo el granizo (Cundinamarca - Colombia). Revista U.D.C.A Actualidad \& Divulgación Científica, 12(2), 79-89. https://doi.org/10.31910/rudca.v12.n2.2009.694

Instituto de Hidrología, Meteorología y Estudios Ambientales. (s.f). Características climatológicas de ciudades principales y municipios turísticos. http://www.ideam.gov.co/documents/21021/418894/Caracter\%C3\%ADsticas+de+Ciudades+Pr incipales+y+Municipios+Tur\%C3\%ADsticos.pdf/c3ca90c8-1072-434a-a235-91baee8c73fc

Fonseca-López, D., Salamanca-López, A. E., Niño-Monroy, L. E., Rodríguez-Molano, C., Hoyos-Concha, J. L., OteroRamírez, I. D., \& Torres Lagos, N. (2019). Caracterización nutricional y de producción de biomasa de Sambucus peruviana, Sambucus nigra y Morus alba en un banco forrajero. Ciencia en Desarrollo, 10(2), 23-32. https://doi. org/10.19053/01217488.v10.n2.2019.9098

Frey, G., Fassola, G., Pachas, N., Colcombet, L., Lacorte, S; Pérez, O., \& Cubbage, F. (2012). Perceptions of silvopasture systems among adopters in northeast Argentina. Agricultural Systems, 105(1), 21-32.

Giraldo, J., Sinisterra, J. A., \& Murgueitio, E. (2011). Árboles y arbustos forrajeros en policultivos para la producción campesina: Bancos Forrajeros Mixtos. LEISA Revista de Agroecología, 27(2), 15-18. http://www.leisa-al.org/web/ index.php/volumen-27-numero-2/1590-arboles-y-arbustos-forrajeros-en-policultivos-para-la-produccion-campesinabancos-forrajeros-mixtos

Gómez, E., Rodríguez, L., Murgueitio, E., Ríos, C., Rosales, M., Molina, CH., Molina, CH., Molina, E., \& Molina, J. (2002). Árboles y arbustos forrajeros utilizados en alimentación animal como fuente proteica. Centro para la Investigación en Sistemas Sostenibles de Producción Agropecuaria.

Gómez, AS., Silva, A., Salazar, J., \& Andrade, J. (2014, 8-10 Outubro). Producción de materia seca y calidad del pasto kikuyo P. clandestinum en diferentes niveles de fertilización nitrogenada nitrogenada y en asocio con aliso Alnus acuminata en el trópico alto colombiano [Conferencia]. 1 Simpósio Internacional de Arborização de Pastagens em Regiões Subtropicais, Embrapa Florestas, Brasil. https://ainfo.cnptia.embrapa.br/digital/bitstream/item/123660/1/p32-41Doc.-268-Anais.pdf

González-Guarín, J. R. (2016). Alternativa silvopastoril para trópico alto con base en bancos forrajeros con Dalias (Dahlia imperialis) y Sauco (Sambucus nigra) en el páramo de Cruz Verde, Ubaque, Cundinamarca, Colombia [Tesis de Maestría, Universidad de Ciencias Aplicadas y Ambientales]. Repositorio de la Universidad de Ciencias Aplicadas y Ambientales. https://repository.udca.edu.co/bitstream/11158/547/1/Tesis\%20dalia\%20final\%202016.pdf 
Grajales-Atehortúa, B. M., Botero-Galvis, M. M., \& Ramírez-Quimara, J. F. (2015). Características, manejo, usos y beneficios del saúco (Sambucus nigra L.) con énfasis en su implementación en sistemas silvopastoriles del trópico alto. Revista de Investigación Agraria y Ambiental, 6(1), 155-168. https://doi.org/10.22490/21456453.1271

Guatusmal-Gelpud, C., Escobar-Pachajoa, L. D., Meneses-Buitrago, D. H., Cardona-Iglesias, J. L., \& Castro-Rincón, E. (2020). Producción y calidad de Tithonia diversifolia y Sambucus nigra en trópico alto andino colombiano. Agronomía Mesoamericana, 31(1), 193-208. https://doi.org/10.15517/am.v31i1.36677

Jaramillo, A. H. (2019). Evaluación de dos especies arbóreas: sauco (Sambucus nigra) y Acacia decurrens) en la alimentación animal. Centro de Biotecnología Agropecuaria. https://repositorio.sena.edu.co/bitstream/handle/11404/5289/sauco_ acacia_en_la_alimentacion_animal.pdf?sequence=4\&isAllowed=y

Jaramillo, C. J. (2006). Evaluación nutricional y agronómica de Morus alba L y Sambucus nigra L y su utilización en alimentación de rumiantes y monogástricos. Revista de Investigación, 6(2), 189-197. https://www.redalyc.org/ pdf/952/95260206.pdf

Jarvis, A., Touval, J., Castro, M., Sotomayor, L., \& Graham, G. (2010). Assessment of threats to ecosystems in South America. Journal for Nature Conservation, 18, 180-188. https://doi.org/10.1016/j.jnc.2009.08.003

Larbi, A., Hassan, S., Kattash, G., Abd El-Moneim, A. M., Jammal, B., Nabil, H., Nakkul, H. (2010). Annual feed legume yield and quality in dryland environments in north-west Syria: 1. Herbage yield and quality. Animal Feed Science and Technology, 160(3-4), 81-89. https://doi.org/10.1016/j.anifeedsci.2010.07.003

Medina, C. (2016). Efectos de la compactación de suelos por el pisoteo de animales, en la productividad de los suelos. Remediaciones. Revista Colombiana de Ciencia Animal, 8(1), 88-93. https://doi.org/10.24188/recia.v8.n1.2016.229

Murgueitio, E., Calle., Z; Uribe, F., Calle, A., \& Solorio, B. (2011). Native tres and shrubs for the productive rehabilitation of tropical cattle ranching lands. Forest Ecology and Management, 261(10), 1654-1663. https://doi.org/10.1016/j. foreco.2010.09.027

Navas, A. (2010). Importancia de los sistemas silvopastoriles en la reducción del estrés calórico en sistemas de producción ganadera tropical. Revista de Medicina Veterinaria, 19, 113-122. http://www.scielo.org.co/pdf/rmv/n19/n19a10.pdf

Navas, A., \& Montaña, V. (2019). Comportamiento de Tithonia diversifolia bajo condiciones de bosque húmedo tropical. Revista de Investigaciones Veterinarias del Perú, 30(2), 721-732. http://dx.doi.org/10.15381/rivep.v30i2.15066

Navas, A., Aragón, F., \& Triana, J. (2020). Efecto del componente arbóreo sobre la dinámica de crecimiento y calidad nutricional de una pradera mixta en trópico alto. Revista de Medicina Veterinaria, 41, 71-82. https://doi.org/10.19052/ mv.vol1.iss 41.7

Portillo, A., Meneses, D., Morales, S., Cadena, M., \& Castro, E. (2019). Evaluación y selección de especies de gramíneas y leguminosas en Nariño, Colombia. Pastos y Forrajes, 42(2), 93-103. http://scielo.sld.cu/scielo.php?script=sci_arttex t\&pid=S0864-03942019000200093

Rivera, L., Armbrechta, I., \& Calle, Z. (2013a). Silvopastoral systems and ant diversity conservation in a cattle-dominated landscape of the Colombian Andes. Agriculture, Ecosystems and Environment, 181, 188-194. https://doi. org/10.1016/j.agee.2013.09.011

Rivera, J. E., Naranjo, J. F., Cuartas, C. A., \& Arenas, F. A. (2013b). Fermentación in vitro y composición química de algunos forrajes y dietas ofrecidas bajo un Sistema Silvopastoril en el trópico de altura. Livestock research for Rural Development, 25(10), Artículo 25174. http://www.lrrd.org/lrrd25/10/rive25174.htm 
Rousseaua, L., Fonteb, S., Téllez, O., Van der, R., \& Lavellea, P. (2013). Soil macrofauna as indicators of soil quality and land use impacts in smallholder agroecosystems of western Nicaragua. Ecological Indicators, 27, 71-82. https://doi. org/10.1016/j.ecolind.2012.11.020

Rueda, O., Cuartas, C., Naranjo, J., Córdoba, C., Murgueitio, E., \& Anzola, H. (2011). Comportamiento de variables climáticas durante estaciones secas y de lluvia, bajo influencia del ENSO 2009-2010 (E1 Niño) y 2010-2011 (La Niña) dentro y fuera de sistemas silvopastoriles intensivos en el Caribe seco de Colombia. Revista Colombiana de Ciencias Pecuarias, 24(3), 512. http://www.scielo.org.co/scielo.php?script=sci_arttext\&pid=S0120-06902011000300032\#7

Sánchez, L., Amado, G., Criollo, P., Carvajal, T., Roa, J., Cuesta, A., \& Barreto, L. (2010). El Saúco (Sambucus nigra L) como alternativa silvopastoril en el manejo sostenible de praderas en el trópico alto Colombia. Corporación Colombiana de Investigación Agropecuaria. https://repository.agrosavia.co/bitstream/handle/20.500.12324/13488/44236_56514. pdf? sequence $=1 \&$ isAllowed $=\mathrm{y}$

Sánchez, S., Crespo, G., \& Hernández, M. (2007). Acumulación y descomposición de la hojarasca en un pastizal de Panicum maximum Jacq. y en un sistema silvopastoril de P. maximum y Leucaena leucocephala. Pastos y forrajes, 30(3), $357-$ 371. http://scielo.sld.cu/scielo.php?script=sci_arttext\&pid=S0864-03942007000300006

Schöbitz, J., Ruiz, M., Balocchi, O. A., Wittwer, F., Noro, M., \& Pulido, R. G. (2013). Effect of increasing pasture allowance and concentrate supplementation on animal performance and microbial protein synthesis in dairy cows. Archivos de Medicina Veterinaria, 45(3), 247-258. https://dx.doi.org/10.4067/S0301-732X2013000300004

Sousa, L., Maurício, R., Moreira, G., Gonc, L., Borges, I., \& Pereira, L. (2010). Nutritional evaluation of Braquiarão grass in association with Aroeira trees in a silvopastoral system. Agroforestry Systems, 79, 179-189. https://doi.org/10.1007/ s10457-010-9297-8

Souza, M. (2003). Contribution of tree to the control of heat stress in dairy cows and the financial viability of livestock farms in humid tropics [Tesis de Doctorado, Centro Agronómico Tropical de Investigación y Enseñanza]. Repositorio del Centro Agronómico Tropical de Investigación y Enseñanza. http://repositorio.bibliotecaorton.catie.ac.cr/handle/11554/965

Tabla-Rojas, J. A. (2019). Efecto del estado fenológico y los niveles de inclusión de Sambucus nigra a la producción de metano [Tesis de maestría, Universidad Nacional de Colombia]. Repositorio de la Universidad Nacional de Colombia. https:// repositorio.unal.edu.co/handle/unal/76880

Vallejo, V. (2013). Importancia y utilidad de la evaluación de la calidad de suelos mediante el componente microbiano: experiencias en sistemas silvopastoriles. Colombia Forestal, 16(1), 83-99. http://www.scielo.org.co/pdf/cofo/v16n1/ v16n1a06.pdf

Van Soest, P. J. (1969). Composition, maturity, and the nutritive value for forages In G. J. Hajny, \& E. T. Reese (Eds.), Cellulases and their applications (Vol. 95, Chapter 16, pp. 262-278). American Chemical Society. https:///doi.org/10.1021/ba1969-0095.ch016

Van Soest, P. J.; Van Amburgh, M. E., \& Tedeschi, L. O. (2000). Rumen balance and rates of fiber digestion. Cornell University. https://www.nutritionmodels.com/papers/VanSoestetalCNC2000150.pdf

Vargas, J., Sierra, A., Mancipe, E., \& Avellaneda, A. (2018). El kikuyo, una gramínea presente en los sistemas de rumiantes en trópico alto colombiano. Revista CES Medicina Veterinaria y Zootecnia, 13(2), 137-156. https://revistas.ces.edu.co/ index.php/mvz/article/view/4558 
Villalobos, L., \& Sánchez, J. M. (2010). Evaluación agronómica y nutricional del pasto ryegrass perenne tetraploide (Lolium perenne) producido en lecherías de las zonas altas de Costa Rica. II. Valor nutricional. Agronomía Costarricense, 34(1), 43-52. https://doi.org/10.15517/RAC.V34I1.6698

Weiss, W. P. (1993). Fiber requirements of dairy cattle: Emphasis NDF. Dairy Science. 